\section{High risk of recurrence in patients with hepatitis-C-related liver cancer}

Patients with hepatitis-C-related hepatocellular carcinoma (HC-HCC) have a higher risk of recurrence after surgery than those with hepatitis-Brelated hepatocellular carcinoma (HB-HCC), according to researchers in Japan. Sasaki et al. conducted a long-term follow-up of patients who underwent surgery for HB-HCC $(n=66)$ or HC-HCC $(n=351)$ between January 1990 and December 1999.

The median follow-up was 11.0 years for patients with $\mathrm{HB}-\mathrm{HCC}$ and 10.1 years for those with HC-HCC. Recurrence occurred in 39 (59\%) patients with HB-HCC and $282(80 \%)$ with $\mathrm{HC}-\mathrm{HCC}$. The odds ratio for risk of recurrence for HC-HCC compared with HB-HCC was 1.93. The 3-year, 5-year and 10-year disease-free survival rates were $57 \%, 54 \%$ and $28 \%$, respectively in the HB-HCC group and $40 \%, 24 \%$ and $12 \%$, respectively in the $\mathrm{HC}-\mathrm{HCC}$ group.

Although the recurrence rates were similar for the two viral groups during the first 2 years of follow-up, the long-term prognosis was considerably worse in patients with $\mathrm{HC}-\mathrm{HCC}$, probably because of a higher frequency of metachronous carcinogenesis in these patients. The authors believe that HCC recurrence in both groups was probably caused by intrahepatic metastasis during the first 2 years. They conclude that the type of viral infection must be taken into account when devising treatment strategies for hepatitis-infected patients with HCC.

Original article Sasaki Y et al. (2006) Risk of recurrence in a long-term follow-up after surgery in 417 patients with hepatitis B- or hepatitis C-related hepatocellular carcinoma. Ann Surg 244: 771-780

\section{Stat5 is a prognostic marker for ER-positive breast cancer}

Members of the signal transducers and activators of transcription (Stat) family have been associated with oncogenesis; constitutively active forms of Stat3 and Stat5 can promote cell proliferation and prevent apoptosis in various cancers. In human breast cancers, nuclear localization and phosphorylation of Stat5 is strongly associated with improved disease-free and overall survival. Yamashita et al. have now demonstrated that Stat5 expression is a prognostic marker of overall survival in patients with estrogen receptor (ER)-positive breast cancer.

The study included 517 breast tumor specimens from patients who had undergone surgical treatment for primary invasive carcinomas. Immunohistochemical analysis showed that Stat3 expression was closely associated with expression of Stat5 $(P<0.0001)$, but there was no association between Stat3 expression and clinicopathological factors. By contrast, Stat5 expression strongly correlated with histological grade $(P<0.0001)$, and was also associated with ER $(P=0.02)$ and progesterone receptor $(P=0.026)$ expression. Although Stat 5 expression was not correlated with disease-free survival, it was associated with an increased overall survival in patients with ER-positive breast cancer $(P=0.0009)$. No association between Stat3 and survival was noted. Moreover, the authors showed that patients with Stat5positive primary tumors responded better to endocrine therapy $(P=0.04)$, and that Stat5 expression was significantly associated with longer survival after relapse $(P=0.0003)$.

The authors conclude that Stat5 is an independent prognostic molecular marker of overall survival in ER-positive breast cancer patients and a predictive factor for endocrine therapy response, indicating that this marker could be used to select patients who might benefit from endocrine therapy.

Original article Yamashita $\mathrm{H}$ et al. (2006) Stat5 expression predicts response to endocrine therapy and improves survival in estrogen receptor-positive breast cancer. Endocr Relat Cancer 13: 885-893

\section{Sunitinib-induced hypothyroidism in patients with gastrointestinal stromal tumors}

The tyrosine kinase inhibitor sunitinib malate is the only drug known to ameliorate survival of patients with nonresectable or metastatic imatinib-refractory gastrointestinal stromal tumors (GISTs). Sunitinib inhibits the mutated kit oncoprotein in GISTs, the receptors for VEGF and PDGF, the FLT3 receptor, and the ret protooncogene expressed in the normal thyroid gland. After the identification of two index case patients with imatinib-resistant GISTs who had developed primary hypothyroidism following sunitinib treatment, a study evaluating thyroid function in patients receiving sunitinib was conducted. 